\title{
6. Revitalisierung der Versdichtung: Das Prosagedicht und Arno Holz' »Revolution der Lyrik«
}

Oftmals lassen sich nur Spekulationen darüber anstellen, warum ein Autor eine bestimmte Textsorte ignoriert oder deren Gebrauch verweigert. Wenn aber die auktoriale Kreation eines neuen Vertextungsmusters als expliziter oder impliziter Gegenentwurf zu einem bereits bestehenden Muster fungiert und zu diesem in Konkurrenz tritt, besteht ein eindeutiger Bezug zum Negierten, welcher die Struktur einer Antwort hat. Ein derartiges negatives Responsionsverhältnis charakterisiert die vielleicht wichtigste, in jedem Fall aber spektakulärste lyrische Innovationsleistung des deutschsprachigen fin de siècle. Arno Holz' 'Erfindung des modernen Mittelachsengedichts nämlich resultiert beileibe nicht nur aus dem Impuls, eine "Revolution der Lyrik (so der Titel einer seiner poetologischen Schriften aus dem Jahr 1899) einzuleiten; sie definiert sich zugleich auch über die Zielsetzung, das als fundamentalen Angriff auf die Grundlagen der Versdichtung verstandene Gattungsmodell des Prosagedichts ein für alle Mal aus dem Zuständigkeitsbereich der Lyrik auszuschließen. ${ }^{1}$ Wohl wissend, daß er sich mit der von ihm vorgeschlagenen Neuerung auf dem Gebiet der Textpräsentation gegen starke, durch kulturell eingeschliffene Rezeptionsgewohnheiten bedingte Widerstände zu behaupten hatte, entwarf der Autor zur Legitimierung seines Vorgehens ein theoretisches Stützgerüst, das - genau betrachtet - ein überaus ehrgeiziges Ziel verfolgt: die Schaffung einer postprosodischen Poetik. Mit seinen Überlegungen knüpft er dabei direkt an die Thesen Max Halbes zur Situation der Versdichtung an, gelangt aber - auch wenn die Zustandsdiagnose beider weitgehend übereinstimmt - zu gegensätzlichen Schlußfolgerungen. Es verwundert denn auch nicht, daß er im Januar 1891 als Redakteur der Freien Bübne für modernes Leben den Abdruck von Halbes Aufsatz Lyrik? verweigert hat, ${ }^{2}$ der ja gerade die »Prosaform [...] als das lyrische Zukunftsideal ${ }^{3}$ postulierte.

1 Fülleboms Ansicht, "daß Holz Vers- und Prosalyrik so eng zusammengeführt hat wie kein anderer«, zeugt dagegen von einem völligen Unverständnis der Poetik dieses Autors; Deutsche Prosagedichte vom 18. Jahrhundert bis zur letzten Jahrhundertwende, S. 276.

2 Vgl. Kapitel III/2.

3 Walter Hettche: Max Halbes Berliner Anfänge, S. 63. Die Frage: »Hatten meine Freunde, die den Vers für die überwundene Form einer überwundenen Epoche erklärten, recht?، (W X, S. 20), die Holz in seinex ersten poetologischen Abhandlung Die Kunst. Ihr Wesen und ibre 
Was Holz mit der naturalistischen Lyriktheorie vorderhand verbindet, ist die scharfe Kritik an einer wängst verlotterten, / abgetakelten Ästhetik" (W X, S. 32), die sich vor allem durch ein überholtes, weil durch automatisierten Gebrauch abgenutztes Repertoire spoetischer Gestaltungsmittel auszeichnet:

Wozu noch der Reim? Der erste, der - vor Jahrhunderten! - auf Sonne Wonne reimte, auf Herz Schmerz und auf Brust Lust, war ein Genie; der tausendste, vorausgesetzt, daß ihn diese Folge nicht bereits genierte, ein Kretin. Brauche ich den selben Reim, den vor mir schon ein anderer gebraucht hat, so streife ich in neun Fällen von zehn den selben Gedanken. Oder, um dies bescheidener auszudrücken, doch wenigstens einen ähnlichen. Und man soll mir die Reime nennen, die in unserer Sprache noch nicht gebraucht sind! Gerade die unentbehrlichsten sind es in einer Weise, da $B$ die Bezeichnung rabgegriffen auf sie wie auf die kostbarsten Seltenheiten klänge. Es gehört wirklich kaum ,Übungr dazu: hört man heute ein erstes Reimwort, so weiß man in den weitaus meisten Fällen mit tödlicher Sicherheit auch bereits das zweite. [...]

Ähnlich die Strophe. Wie viele prachtvolle Wirkungen haben nicht ungezählte Poeten jahrhundertelang mit ihr erzielt! Wir alle, wenn wir Besseres nicht zu tun wissen und alte Erinnerungen locken, wiegen uns noch in ihr. Aber ebensowenig wie die Bedingungen stets die selben bleiben, unter denen Kunstwerke geschaffen werden, genau so ändern sich auch fortwährend die Bedingungen, unter denen Kunstwerke genossen werden. Unser Ohr hört heute feiner. Durch jede Strophe, auch durch die schönste, klingt, sobald sie wiederholt wird, ein geheimer Leierkasten. Und gerade dieser Leierkasten ist es, der endlich raus muß aus unserer Lyrik. Was im Anfang Hohes Lied war, ist dadurch, daß es immer wiederholt wurde, heute Bänkelsängerei geworden! (W X, S. 498-500)

Holz erklärt deshalb mit radikaler Geste alle süberlieferten Kunstmittel« (W X, S. 498) wie Metrum ${ }^{4}$, "Reim, Strophe, Parallelismus, Alliteration und Assonanz« (W $\mathrm{X}, \mathrm{S} .510$ ) für obsolet. Doch auch die freirhythmische Dichtung kann nach seiner Einschätzung keine unlimitierte literarische Ausdrucksfreiheit gewährleisten, weil sie auf Grund ihrer Gattungstradition an stilistische und rhetorische Konventionen gebunden ist. Ihre Lexik etwa sei, wie einschlägige Gedichte von Goethe oder Heine zeigten, generell auf »Pathos« (W X, S. 501) gestimmt. Damit aber werde zwangsläufig wein vorgefaßtes Klangschemaa (W X, S. 493) erzeugt, das die scheinbar freien Rhythmen letztlich zu einem "Konglomerat von metrischen Reminiszenzen« (W X, S. 537) mache. Und dort, wo das Versgedicht sich seines spoetischen Charakters vollständig entschlage, werde die Lyrik als eigenständiger sprachlicher Modus insgesamt suspendiert. ${ }^{5}$

Gesetze (1891) aufwirft, ist denn auch als direkter Reflex auf die Herausforderung durch Halbes Thesen zu werten.

Der Autor ist sich dabei der Tragweite seines Handelns wohlbewußt, sieht er doch mjede Wortkunst, von frühester Urzeit bis auf unsere Tage«, mauf Metrik« als »letztem, tiefstuntersten Formprinzip [...] gegründet" (W X, S. 472).

5 Liliencrons freirhythmische Texte beispielsweise erscheinen Holz als »reine Prosan (W X, S. 619). So kommt ihm das Versgedicht Betrunken (Erstdruck 1892) wwie der Anfang einer Skizze« vor: "Stelle ich mir vollends auch noch dic Schreibweise [gemeint ist offenbar: das 
Die Prosa aber galt Holz spätestens seit Mitte der neunziger Jahre als defizientes Ausdrucksregister, weil sie Sprache in unkünstlerischer Anarchie gebrauche und wsich um Klangwirkungen überhaupt nicht» (W X, S. 538) kümmere. Den Roman etwa qualifizierte er umstandslos als ästhetisch minderwertige "Zwitterform « $a b,{ }^{6}$ aber auch das wsogenannte Prosadrama» erschien ihm als beklagenswertes Resultat einer verhängnisvollen "Auflösung« der "Wortkunst" (W X, S. 472): "Alle neuere Epik und Dramatik war zu Prosa zerfallen« (W X, S. 492, Anm.), lautet 1898 das ernüchternde Fazit. ${ }^{7}$ Holz' Hoffnung richtete sich daher auf eine Neubegründung der Versdichtung, einen literarischen Modus, den er im Gegensatz zu den meisten seiner naturalistischen Kollegen gerade nicht als historisch obsolet gewordenes Gestaltungsmuster ansah, sondern statt dessen zum Residuum der >Poesiec erklärte: "Alle Kunst war mir Poesie und alle Poesie Lyrik.« (W X, S. 489) ${ }^{8}$ Dementsprechend wandte er sich nach seinen in der kurzen Phase der Zusammenarbeit mit Johannes Schlaf (1888-92) unternommenen "Prosaexperimenten« (W X, S. 504) ${ }^{9}$ ausschließlich dem Drama und der Lyrik $z^{10}$ - jenen Literaturformen also, die auf Grund der »knappen Kondensiertheit" (W X, S. 37) ihrer Sprache allen anderen Gattungen überlegen schienen. Und weit davon entfernt, die Versdichtung sprosaisieren zu wollen, suchte er vielmehr nach einem Weg, sie - zeitgemäß erneuert - grundlegend und endgültig zu restituieren. Eindrucksvollster Beleg dieses Bestrebens ist der aus

Druckbild] so vor, so wird diese Illusion vollständig. Ich verspüre dann nicht mehr auch nur noch den geringsten Rhythmus; wie ich ihn in jeder anderen Prosa, trozdem er in ihr drinsteckt, auch nicht verspüre.« (W X, S. 620) Selbst Walt Whitmans kühne, die Verszeile sprengende Lyrik gelange über »einen Mischmasch aus freiem Rhythmus und Prosa (W X, S. 539) nicht hinaus. Den Grund dafür benennt Holz in einem Brief an Eduard Bertz vom 28. Februar 1907. Dort heißt es, eine Aussage von O.E. Lessing zustimmend zitierend: "Whitmans Form ist immer ein monotones Kirchenrezitativ: ob er von einer Reiterattacke spricht oder vom Wesen des 'Höchsten«; Amo Holz: Briefe. Eine Auswahl. Hrsg. von Anita Holz und Max Wagner. Mit einer Einfühnung von Hans Heinrich Borcherdt. München: Piper 1948, S. 173.

6 Schon in seinem frühen Essay Die Dichtkunst der Jetrtzeit hatte Holz die Ansicht vertreten, der Roman könne als weigene Kunstform nicht anerkannt werden - eine Position, von der er aber zwischenzeitlich wieder abgenückt war; zitiert nach Helmut Scheuer. Amo Holz im literarischen Leben des ausgehenden 19. Jahrhunderts (1883-1896), S. 90.

7 Schon im Buch der Zeit (1885) hatte es geheißen: „Die Welt ist heute prosatollk (W I, S. 27).

8 Burdorf konstatiert bei Holz denn auch eine wKonzentration seiner Poetik auf die Lyriktheorie«; Dieter Burdorf: Poetik der Form. Eine Begriffs- und Problemgeschichte, S. 374.

9 Diese finden sich in den beiden Bänden Papa Hamlet (1889) und Nexe Gleise (1892).

"Lyrik und Drama [...] haben sich formal wieder zu einer Einheit geschlossen!« (W X, S. 473) bemerkt Holz später im Hinblick auf seine eigene literarische Produktion. Sprechender Beleg dafür ist die Tatsache, daß »Holz eine wichtige Partie des Ignorabismus, die Rede Domingers von der Gerechtigkeit aus dem IV. Akt, wörtlich und nur auf die Mittelachse umgestellt, in den Phantasus von 1916« übernahm; Karl Geisendörfer: Die Entwicklung eines lyrischen Weltbildes im Phantasus von Amo Holz. In: Zeitschrift für deutsche Philologie 82 (1963), S. 240. 
vielen Einzeltexten bestehende, dennoch zu einem lyrischen Gesamtgebilde zusammengeschlossene Gedichtkomplex Pbantasus, der 1898/99 zuerst in zwei schmalen Heften mit einem Umfang von etwas über 100 Seiten erschien und ständig modifiziert und erweitert wurde, bis er in der Werkausgabe von 1925 auf insgesamt 1345 Seiten angewachsen war, die auf drei ebenso großformatige wie voluminöse Bände verteilt werden mußten. ${ }^{11}$

Um nun den Geltungsbereich der Lyrik ein für allemal zu sichern, benötigte Holz ein Strukturierungsprinzip, das sich von den bisherigen poetischen Gestaltungsmitteln grundlegend abhob. Er fand es schließlich im Rhythmuss, und zwar im mnatürlichem (W X, S. 502), den er kurzerhand an die Stelle des Metrums setzte. ${ }^{12}$ Metrik und Rhythmik sollen sich dabei zueinander verhalten wie sAußen zu >Innen ${ }^{13}$ wie „Willkür« zu »Notwendigkeit«. ${ }^{14}$ Worauf Holz abzielte, war »eine Lyrik, die auf jede Musik durch Worte als Selbstzweck verzichtet und die, rein formal lediglich durch einen Rhythmus getragen wird, der nur noch durch das lebt, was durch ihn zum Ausdruck ringt« (W X, S. 494). Die herkömmliche Verslyrik schien ihm dafür gänzlich ungeeignet, weil sie den literarischen Ausdruck von vornherein reglementiere:

Jeder Wortkünstler bisher fand zwischen sich und dem, was er ausdrücken wollte, bereits immer etwas vor. Ein formales, metrisches Schablonengebilde, das er entweder glatt akzeptierte, oder höchstens [...] minimal varierte! In eine ihm überlieferte Form preßte er willkürlich seinen Inhalt, statt umgekehrt, wie ich dieses verlange, die erst gesuchte, noch gar

11 Aus dem Nachlaß wurde schließlich noch eine weitere Fassung des "Riesen-PhantasusNonplusultra-Poems« (Bd. 1, S. 454) von 1584 Seiten Umfang publiziert, vgl. Amo Holz: Werke. Hrsg. von Wilhelm Emrich und Anita Holz. Bd. 1 bis 3. Neuwied/Berlin: Luchterhand 1961.

12 Interessanterweise berief sich ja auch Schlaf (vgl. Kapitel III/4b) bei der von ihm propagierten »Revolution der lyrischen Form» auf den matürlichen Rhythmus«; Johannes Schlaf: Neue Lyrik, S. 168. Was ihn von Holz unterscheidet, ist der unterschiedliche Stellenwert des wahmehmenden Subjekts: Während Schlaf dem "Rhythmus der gesteigerten Nervenvibrationen « nachspürt, dem er einen genuinen Eigenwert zuschreibt, sucht Holz Rhythmizität ontologisch zu verankem, was das empfindende Ich zweitrangig, wenn nicht überflüssig werden läßt; ebd.

Nach eigenem Bekunden ging es dem Autor darum, die - im Zuge seiner Rhythmustheorie postulierte - sinnere Melodieu der Worte mnach außen zu projizieren« (W X, S. 657). Die Rede von der minneren Rhythmiku (W X, S. 645) eines Textes enweist sich bei genauerer Betrachtung als unbewußte Neuakzentuierung des Konzepts der sinneren Form und reiht $\mathrm{Holz}$ in jene Gruppe von Autoren der Jahrhundertwende ein, die sich unter Rückgriff auf dieses Postulat eine Erneuerung der Dichtkunst versprachen (vgl. hierzu Kapitel III/7).

Holz charakterisiert Rhythmiks als upermanente, sich immer wieder aus den Dingen neu gebärende, komplizierteste Notwendigkeit» und setzt sie nachdrücklich ab von der Metrik, die er als "primitive, mit den Dingen nie, oder nur höchstens ab und zu, nachträglich und wie durch Zufall koinzidierende Willkür« (W X, S. 472f.) bezeichnet. Daneben denkt er das Verhältnis beider als eines der Inklusion, wobei Rhythmik der "Überbegriff (W X, S. 650) sei, der auch - gewissermaßen als Grenzfall - das schmale Gebiet der Metrik beinhalte. 
nicht vorhandene Form aus seinem Inhalt unwilikürlich, dafür aber um so notwendiger erst wachsen zu lassen. (W X, S. 640) ${ }^{15}$

Anstatt daß wsich Inhalt und Form« wie in Gedichten der traditionellen Machart nur weinen flüchtigen Augenblick einmal decken«, sollte fortan wdie permanente Kongruenz dieser beiden « (W X, S. 621) Aspekte des Sprachkunstwerks erreicht werden. ${ }^{16}$ Diese werde von den vorgestanzten Gestaltungsmitteln der Prosodie ebenso verfehlt wie von der alle Unterschiede einebnenden Prosa. Traditionelle Verslyrik und ungebundene Sprache seien also gleichermaßen Modi, die einen wirklich sfreien, d.h. individuellen literarischen Ausdruck verhindem.

Damit Inhalt und Form vollständig zur Deckung gebracht werden konnten, mußte der geheimnisvolle matürliche« Rhythmus freilich außerästhetisch fundiert werden. Deshalb suchte Holz - ebenso wie Dauthendey und Schlaf - die Ästhetik direkt an die moderne Naturforschung anzukoppeln, ${ }^{17}$ um so seiner Kunsttheorie einen legitimierenden Rahmen zu verschaffen. ${ }^{18}$ Faktisch orientierte er sich dabei - genau wie seine beiden Kollegen auch - im wesentlichen an den Theoremen der vitalistischen Populärwissenschaft seiner Zeit. Während Dauthendey aus monistisch akzentuierter Atomistik einerseits, fechnerscher Psychophysik andererseits und Schlaf vornehmlich aus der haeckelschen Entwicklungslehre die Schlußfolgerung ableiteten, das literarische Ausdrucksspektrum so variabel wie möglich zu halten und alle Arten von formaler Entgrenzung zu erproben, konstruierte Holz eine spekulative Naturphilosophie, die ihm ein festes ontologisches Fundament bot, von dem aus die Lyrik neubegründet werden konnte. Das Verhältnis von Kunst und Natur denkt er in einer Art psychophysischen Entsprechungslehre kurzerhand als eines von Mikrokosmos zu Makrokosmos: Rhythmizität wohne demnach bereits den sDingen

15 Zugrunde liegt diesen Überlegungen die berühmte, in der Schrift Die Kunst. Ihr Wesen und ihre Gesetze (1890/91) erstmals entwickelte Formel: "Kunst = Natur - Xu (W X, S. 80).

16 Die von Holz geforderte "Einheit [...] von Form und Inhalt" (W X, S. 645) werweist sich unter dieser Perspektive als unbewußte Neufassung von Goethes Konzept der inneren Form, und sie leidet unter dem Grundproblem der Vorstellung einer inneren Form, daß sie nicht anhand objektivierbarer Kriterien überprüft werden kann, da der Aspekt der äußeren Form als unangemessen und sekundär abgetan wird«; Dieter Burdorf: Poetik der Form. Eine Begriffs- und Problemgeschichte, S. 384. Vgl. Kapitel III/7.

17 Sprengel konnte sogar nachweisen, daß Holz in seinem Phantasus mehrfach Abschnitte aus Wilhelm Bölsches Entnicklungsgeschichte der Natur (1894/96) paraphrasiert hat, vgl. Peter Sprengel: Darwin in der Poesie. Spuren der Evolutionslehre in der deutschsprachigen Literatur des 19. und 20. Jahrhunderts. Würzburg. Königshausen \& Neumann 1998, S. 25-30.

Wie Holz in einem Brief an Richard Schaukal vom 13. Mai 1902 bekräftigt, seien in seinen Schriften msämtliche Termina [sic]« mim naturwissenschaftlichen" und micht in irgendeinem moralisch-ästhetischen Sinne» zu verstehen; Amo Holz: Briefe, S. 129. Und noch in der Neuen Wortkunst (1925) beruft er sich auf John Stuart Mills Gedanken »von der durchgängigen Gesetzmäßigkeit alles Geschehens» (W X, S. 63f.) und zollt damit - zumindest rhetorisch - dem vermeintlich kausalmechanistischen Weltbild der Naturwissenschaften Tribut. 
selbst inne. Dem Künstler kommt in diesem Zusammenhang nurmehr die Aufgabe zu, den Rhythmus als gleichsam naturhafte Qualität in den Bereich der Ästhetik zu übertragen. Er erzeugt ein sunmittelbar aus dem Erleben entstehendes Sprachelement, das als Palimpsest die Sprache des Ich und der Dinge zugleich lesen läßt« ${ }^{19}$. Wenn die künstlerische "Vorstellung« nur »klar« genug sei, ergebe sich »die Form aus ihr geradezu von selbst« (W X, S. 547).

Ein derart essentialistisches Verständnis ästhetischer Termini aber tendiert dazu, Kunst nicht nur aus ihren sozialen und kulturellen Rahmenbedingungen herauszulösen, sondern auch ihre Historizität zu leugnen. Indem Holz eine verborgene shythmischer Grundstruktur der Wirklichkeit postuliert, die es sprachlich nur freizulegen gelte, stellt er Geschichte kurzerhand still. ${ }^{20}$ Damit nicht genug: Die Konstruktion einer unverrückbaren ontischen Bezugsgröße degradiert auch Autor wie Leser als subjektive Einflußfaktoren literarischer Wirkung ${ }^{21}$ zu gleichsam akzidentiellen Faktoren. Artifiziell konstruierte Wort-Kunst wird so wieder auf 'Natur` zurückgeführt, ${ }^{22}$

19 Ingrid Strohschneider-Kohrs: Sprache und Wirklichkeit bei Amo Holz. In: Poetica 1 (1967), S. 59. Burdorf bemerkt in diesem Zusammenhang. »Holz geht [...] auch für den Bereich der Kunst ganz ungebrochen von einer Abbildtheorie der Sprache aus; jede Vokabel hat für ihn ihr reales Äquivalentı. Dieter Burdorf: Poetik der Form. Eine Begriffs- und Problemgeschichte, S. 376.

20 Daß die ohnehin nur schwach ausgeprägten soziologischen Elemente von Holz' Kunsttheorie im Lauf der Zeit gänzlich durch naturphilosophische Spekulation verdrängt werden, übersieht Hechler; vgl. Manfred Hechler: Die soziologische Dimension der Kunsttheorie von Amo Holz. Frankfurt a.M./Bem: Lang 1981 (= Helicon 2).

21 Holz übernimmt sogar Elemente der sog. Ohrenphilologie (Ferdinand Saran, Eduard Sievers) seiner Zeit, die intensiv an der vollständigen rhythmischen Analyse des künstlerischen Wortmaterials arbeitet. So paraphrasiert er etwa das "Klangschemau der Zeilen "Vor meinem Fenster / singt ein Vogel. // Still / höre ich zu. // Mein Herz ... vergeht. // Er singt, / was ich als Kind ... so ganz besaß / und dann ... vergessen! « durch folgende numerische Auflistung: $21131 / 3131 / / 3 / 2113 / / 13-13 / / 13 / 2113-1313 / 13$ 131 « (W X, S. 550), wobei die Ziffern die Stärke der jeweiligen Silbenbetonung andeuten sollen. Dies stelle freilich nur eine grobe Annäherung an die tatsächliche phonetische Struktur des Textes dar, denn »daß man im übrigen, falls man Gewicht darauf legt, die einzelnen Tonwerte noch differenzierter darstellen kann, ist selbstverständlich» (ebd.). Heuslers zwar imponierende, aus heutiger Sicht aber zugleich hoffnungslos antiquiert anmutende Taxonomie des Verses ist übrigens ein später Ausläufer dieses methodischen Ansatzes; vgl. Andreas Heusler. Deutsche Versgeschichte mit Einschluß des altenglischen und altnordischen Stabreimverses. 3 Bde. Berlin/Leipzig: de Gruyter 1925-29 (= Grundriß der germanischen Philologie 8). Die Einsicht, daß jedes sprachliche Denotat ein Spektrum von Möglichkeiten performativer Artikulierung zuläßt, vermochte sich gegen die auf Vollständigkeit und Eindeutigkeit ausgerichteten Systematisierungsbestrebungen der älteren Metrikforschung erst allmählich durchzusetzen.

22 Schon Emrich konstatiert "Amo Holz hat theoretisch wie dichterisch versucht, die Kunst wieder in Natur zu verwandeln."Wilhelm Emrich: Zur Ästhetik der modemen Dichtung. In: W. E.: Protest und Verheißung. Studien zur klassischen und modernen Dichtung [1954]. Frankfurt a.M./Bonn: Athenäum 1960, S. 129. Holz selbst hat seine »Revolution 
und verstärkte Arbeit an der Sprache (wie sie etwa in der gewaltigen Aufschwemmung des Phantasus-Projekts zum Ausdruck kommt) ${ }^{23}$ steht lediglich im Dienst der - tendenziell unabschließbaren - Aufgabe, die Variable sx، aus Holz' berühmter Formel konsequent zu minimieren:

If we [...] relate Holz's particular prescription for poetic form to his more general definition of art (K = N $-X:$ : Die Kunst hat die Tendenz, die Natur zu sein, Sie wird sie nach Maßgabe ihrer Mittel und deren Handhabungi), it is clear that the importance of rhythm for Holz lies in the fact that potentially it enables the artist to reduce one of the $\mathrm{x}$ ( factors (namely the handling of the artistic means) to the process of divesting language of the weight of conventional usage in order to reveal its intrinsic significance ${ }^{24}$.

Und auch wenn er zugesteht, daß jeder Künstler den dem «Stoff“ angemessenen Rhythmus »durch seine Individualität variiert« (W X, S. 549), ${ }^{25}$ geht es dem Autor letztlich doch um die Reduzierung des subjektiven Faktors in der Ästhetik - gemäß der Maxime: „Determination, auch hier, und nicht mehr, wie bisher, sogenannte ,Willensfreiheitd« (W X, S. 645f.)

$\mathrm{Daß}$ er tatsächlich auf die »möglichste Ausschaltung« des mIchs« (W X, S. 662) abzielt, ${ }^{26}$ verdeutlichen im übrigen auch seine Anstrengungen um eine Strukturierung seines lyrischen Hauptwerks nach arithmetischen Aspekten. ${ }^{27}$ So äußert Holz in dem Aufsatz Idee und Gestaltung des Phantasus (1918), daß seiner "Rhythmik als allerletztes ein bestimmtes Zahlenverhältnis zugrunde liegt« (W X, S. 660). Die komplizierte "Zablenarchitektonik«, die er für sein Werk entwirft, ${ }^{28}$ ergebe sich dabei wzwingend aus

der Lyrik« als geschichtlich notwendigen Versuch gedeutet, endlich »den großen Weg zur Natur zurïck (W X, S. 489) zu beschreiten.

In einem Brief an Franz Servaes vom 6.1.1917 unterstreicht Holz selbst, daß seine langjährige Revisionsarbeit im Dienste sprachlicher "Differenzierungu stehe; Amo Holz: Briefe, S. 233. Rob Bums: The Quest for Modemity: The Place of Amo Holz in Modem German Literature. Frankfurt a.M./Bern: Lang 1981 (= Europäische Hochschulschriften I/431), S. 150. Wenn dem aber tatsächlich so wäre, dann könnte nicht von einer universalen Geltung von Holz' sog. Kunstgesetz gesprochen werden, weil die jeweils individuellen Ergebnisse dann intersubjektiv nicht mehr vermittelbar wären.

Rappl hat als erster gezeigt, daß dies mit einer Selbstpreisgabe des Subjekts einhergeht; vgl. Hans-Georg Rappl: Die Wortkunsttheorie von Amo Holz. Diss. (Masch.) Köln 1957. Emrich hat diesen Gedanken aufgenommen und auf die Formel gebracht „Ein „Esı, bzw. die NNatur wird Dichter der Dichtung. nur so kann die notwendige Form aus der Sache entspringenc, Wilhelm Emrich: Amo Holz und die modeme Kunst. In: W. E.: Protest und Verheißung. Studien zur klassischen und modemen Dichtung [1954], S. 158. Burdorf stellt zutreffend fest: „Holz schwankt [...] zwischen einem organologischen Modell, das das Gedicht zu einem ideellen Gesamtkörper erklärt und in den späteren PhantasusFassungen immer dominanter wird, und einem vechnizistischen Modell, das aus der Vorstellung von einem quasi-naturgesetzlichen Kunstgesetz Regeln für die Form literarischer Texte ableiten zu können beansprucht. « Dieter Burdorf: Poetik der Form. Eine Begriffs- und Problemgeschichte, S. 384. 
sich selbst und ohne jedes [...] Dazutun ( W X, S. 662) seitens des Künstlers. Indem er solcherart die seit jeher enge Verwandtschaft zwischen Mathematik und Musik zu Legitimationszwecken ausbeutet, ${ }^{29}$ entsteht eine logisch verbrämte "Zahlenmystik" (W X, S. 663), ${ }^{30}$ die nach und nach an die Stelle der traditionellen Poetik tritt. Weshalb Holz auf ein solches Konstrukt zurückgreift, liegt auf der Hand: Weil eine ontologisch ausgerichtete, mathematisch grundierte Rhythmuslehre schlechterdings walles umfaßt und absolut nichts mehr außerhalb der Grenzen ihrer Greifmöglichkeit liegt«, garantiert sie eine »noch tiefere, gewaltigere und noch zwingendere Bindung" (W X, S. III) der Literatursprache als sie die bisherige, nur vermeintlich sgebundener Rede bieten kann, deren Regeln sich letztlich nur aus jahrhundertealten Konventionen herleiten. ${ }^{31}$ Nichts kann mithin eine wirkungsvollere naturwissenschaftlich-philosophische Letztbegründung von >Poesier liefern als die Annahme eines rhythmisch strukturierten ontischen Realitätssubstrats. ${ }^{32}$

Auch die formalen Neuerungen, die Holz in die Lyrik einführte, dienen letztlich dem Zweck, die überzeitliche Suprematie der Versdichtung ein für allemal zu sichern. ${ }^{33}$ Sichtbar wird dies freilich erst, wenn man seine Verfahrensweisen im Kontext der Formexperimente und Gattungsinnovationen der Jahrhundertwende be-

haltsmoment, von den kleinsten bis zu den größten Stoffkomplexen, genau abmeßbare Zahleneinheiten zugeordnet werden. Diese Zahleneinheiten - meist ist ihre Grundwurzel eine 3 - bestimmen dann in einer sppringenden Reihe, z.B. 3-5-7-12 usw. die Silbenanzahl eines Wortes, die Wortanzahl eines Gesäzzels< [ - also eines durch vorausgehende und nachfolgende Leerzeilen als rabgeschlossen markierten Syntagmas, das der konventionellen Strophe entspricht -] und dann noch die Anzahl der Gesätzel, die ein Gedicht enthält.« Karl Geisendörfer: Die Entwicklung eines lyrischen Weltbildes im Phantasus von Arno Holz, S. 247. Holz' Grundgedanke wurde dann von seinem Schüler Robert Reß in der Schrift Die Zabl als formales Weltprinzip (1926) systematisch ausgefaltet. In einem Brief an Wolfgang Schumann vom 27. März 1926 bezeichnet Holz die "Zahlenarchitektonik« seines Phantasus auch als mklingende Mathematikw; Amo Holz: Briefe, S. 265. In diesem Zusammenhang ist daran zu erinnem, daß schon Bölsche die "Dichtungu der Zukunft als meine Art von Mathematik» bezeichnet hat; Wilhelm Bölsche: Die naturwissenschaftlichen Grundlagen der Poesie. Prolegomena einer realistischen Ästherik, S. 25. Schulz spricht in diesem Zusammenhang von einer mmystifizierten mathematischen Poetike; Gerhard Schulz: Amo Holz. Dilemma eines bürgerlichen Dichterlebens. München: C.H. Beck 1974, S. 233.

31 „Was mir [...] vorschwebte, war eine noch weit umfänglichere Sprachbindung als die bisherige gewesen!" (W X, S. 492f., Anm.), gesteht Holz unumwunden. An anderer Stelle spricht er auch vom »Formgeista (W X, S. 636), der ihn bei seiner Revision der Poetik geleitet habe. Mit der Annahme präformierter, gleichsam naturwüchsiger Formen greift er auf Ansätze der Ästhetiktheorie des 18. Jahrhunderts - nicht zufälig zitiert Holz an einer Stelle seiner Neuen Wortkunst zustimmend Herder (vgl. W X, S. 580f.) - zurück und amalgamiert sie mit Versatzstücken der Evolutionsbiologie, die zu einer "monistischen Allphilosophie» ausgebaut wird; Gerhard Schulz: Arno Holz. Dilemma eines bürgerlichen Dichterlebens, S. 210. Das ist insofern bedeutsam, als eben sie es sind, welche letztlich die Bedeutung des Autors für die Entwicklung der Lyrik und damit seinen Rang in der Literaturgeschichte ausmachen. 
trachtet. Das auffälligste Merkmal von Holz' „Revolution der Lyrik stellt sicher die prima vista wetwas sonderbar anmutende Druckanordnung« seiner Texte dar: "Unregelmäßig abgeteilte Zeilen« werden typographisch um eine »unsichtbare Mittelachse» (W X, S. 503) angeordnet. Diese Innovation, die der Autor selbst als ästhetischen Durchbruch empfand, hat schon bei den Zeitgenossen Unverständnis und Belustigung hervorgerufen, weil sie als lediglich arbiträre optische Darbietungsform wahrgenommen wurde, die den generischen Status eines Textes in keiner Weise tangiere. Und auch in der Forschung wurde Holz' Vorstoß im allgemeinen als der Entstehungszeit geschuldeter Tribut an die ornamentalen Vorlieben der Jahrhundertwende verbucht - eine Deutungsperspektive, die freilich mehr vom äußeren Erscheinungsbild (der dekorativen Schrifttype der Pbantasus-Erstausgabe und der von Fritz Rumpf im Sinne der Jugendstilästhetik entworfenen Titelvignette) als von der eigentlichen Logik der Textgestaltung ausgeht. ${ }^{34}$ In der Tat bietet ja die Mittelachsengliederung, nüchtern betrachtet, gegenüber der herkömmlichen linksbündigen Anordnung der Verszeile keinen wirklichen Zugewinn an Ausdrucksmöglichkeiten. Holz' typographische Neuerung bringt sogar den Nachteil mit sich, daß Verse, welche die Zeilengrenze überschreiten, nicht mehr darstellbar sind - ein Manko, das der Autor dadurch zu kompensieren suchte, daß er den Satzspiegel enorm vergrößerte. ${ }^{35}$ "Zieht man« schließlich

in Betracht, daß bei der zentrierten Anordnung ungleichmäßig langer Verse der linke Anfang jeder Zeile immer neu gesucht werden muß, so wird deutlich, daß Mittelachsengedichte den Lesekomfort und die Lesegeschwindigkeit eher herabsetzen. Die besondere graphische Anordnung fördert also die Segmentierung des Gedichts in seine einzelnen Zeilen, während der Übergang von einer Zeile zur nächsten eher erschwert wird. ${ }^{36}$

34 Gleichwohl sind natürlich Experimente mit der Schrift und ihrer Materialität ein Signum der Jahrhundertwende; vgl. in diesem Zusammenhang Friedrich A. Kittler: Aufschreibesysteme 1800/1900 [1985]. 2., erweiterte und korrigierte Auflage. München: Fink 1987, S. 257-270. Veit mißversteht freilich deren Verfahrenslogik, wenn sie die drucktechnischen Innovationen Georges und Holz' simplifizierend als Indiz für die Wiederbelebung einer strengen Form deutet: "Dic Betonung der Form begann bereits in den späten neunziger Jahren mit äußeren Merkmalen: z.B. der ausgeprägt individuellen Interpunktion und Orthographie Stefan Georges und seiner Drucktypen oder dem Mittelachsensystem des Phantasus (1898/99) von Amo Holz.« Elisabeth Veit: Fiktion und Realität in der Lyrik. Literarische Weltmodelle zwischen 1890 und 1918 in der Dichtung Max Dauthendeys, Richard Dehmels und Alfred Momberts, S. 139, Anm. 1.

35 Dies ändert aber nichts daran, daß vor allem die späteren Phantasus-Texte in kleinformatigen Büchern und vielen Zeitschriften oft nicht mehr adäquat präsentiert werden konnten. Als etwa in den zwanziger Jahren eine Anthologie vorbereitet wurde, in denen auch Lyrik von ihm abgedruckt werden sollte, "verlangtes $\mathrm{Holz}$ nicht nur eine genaue "Formatangabe" und eine "Typenprobe«, sondem vor allem auch die "Zusicherung, daß keine Verszeile umbrochen werdew; zitiert nach Helmut Scheuer. Arno Holz im literarischen Leben des ausgehenden 19. Jahrhunderts (1883-1896), S. 299, Anm. 135. 
Man mag deshalb verwundert sein über die Diskrepanz, die zwischen der faktischen Leistung von Holz' Notationssystem und seiner übertrieben vollmundigen Selbstdarstellung besteht. Die Verve jedoch, mit welcher der Autor sein typographisches Modell anpries $^{37}$ und lebenslang gegen alle Anwürfe von außen verteidigte, gibt $z u$ denken, sofern man sie nicht einfach als Ausdruck naiver Verblendung oder rechthaberischen Starrsinns abtun will. Fragt man also nach der eigentlichen Leistung seiner Innovation, so wird man sie offenbar nicht in der Steigerung, sondern paradoxerweise gerade in der Begrenzung von Darstellungsoptionen suchen müssen. So preziös das gewählte Druckbild zunächst erscheinen mag, es wirkt zumindest auf den unvoreingenommenen Rezipienten fraglos deautomatisierend, ${ }^{38}$ weil er einen Text wahrnimmt, der sich, obgleich er unverkennbar an die optischen Merkmale herkömmlicher Lyrik erinnert, doch davon auch deutlich abhebt. Holz entzieht nämlich die Grenzen des Satzspiegels tendenziell der Wahrnehmung durch den Leser. Während der Blocksatz der Prosa ständig die sRänderr des Textes vor Augen führt, und die verbindliche linksbündige Druckanordnung herkömmlicher Versdichtung zumindest den Zeilenanfang durch die - innerhalb gewisser Toleranzen - variable Extension des Textes auf der rechten Seite besonders betont, kappt die Mittelachsengliederung diese partielle typographische Übereinstimmung der Lyrik mit der Prosa und setzt an deren Stelle ein trennscharfes Darbietungsmodell.

Damit reagiert der Berliner Autor präzise und direkt auf die mit der Einführung von Vers/Prosa-Mischformen in der deutschen Literatur verbundene Aushöhlung des bisherigen dichotomisch verfaßten Gattungssystems. Wie sehr Holz' Neubegründung der Lyrik die konkurrierenden Gestaltungsformen des Prosagedichts bzw. der spoetischen Prosas im Blick hat, zeigt sich nicht zuletzt daran, daß er in seinem ästhetischen Grundlagenwerk, der Befreiten Worteunst, ausführlich auf prominente Ausprägungen dieser Gattungsmodelle wie Jean Pauls "Streckverse« oder Walt Whitmans prosanahe Langzeilendichtung eingeht (vgl. W X, S. 582-585) und - in Reaktion auf literarische Kritiken - auch Pierre Louys' Chansons de Bilatis und Anna Croissant-Rusts Gedichte in Prosa erwähnt (vgl. W X, S. 614 und 617). ${ }^{39}$ Daneben kannte er

selbst hatte die Wahl der Mittelachsengliederung ja mit ophthalmoökonomischen Argumenten gerechtfertigt (vgl. W X, S. 574) - eine Scheinbegründung, der noch Kittler Glauben schenkt; siehe Friedrich A. Kittler: Aufschreibesysteme 1800/1900 [1985], S. 230.

37 So beteuert er etwa in seiner Selbstanzige (1898), daß er ssich mit aller Energie [...] jahrelang [...] das Gehirn zergrübelt« und erst, machdem ein Resultat dabei herausgesprungen " (W X, S. 505) sei, den Versuch unternommen habe, es literarisch umzusetzen.

Interessanterweise nehmen Holz' kunsttheoretische Schriften mehrfach Einsichten vorweg, die dann von den russischen Formalisten genauer analysiert worden sind; der Mechanismus der Automatisierung stellt wohl das beste Beispiel hierfür dar.

39 Auch Holz' Adepten haben diesen Zusammenhang wahrgenommen. So enthält etwa Paul Emsts Lyriksammlung Pofymeter (1898), die kurz vor der ersten Ausgabe des Phantasus erschien und schon im Titel auf Jean Pauls "Streckverse" anspielt, mehrere Gedichte, die Holz' Theorie der Mittelachsengliederung umsetzen. Die Texte sind aus dem engen per- 
mit Sicherheit auch die in der Öffentlichkeit z.T. enthusiastisch rezipierten lyriknahen Prosatexte seines ehemaligen Mitstreiters Johannes Schlaf. ${ }^{40}$

Holz war also mit der Problematik der Codierung von Poetizität genauestens vertraut und entwickelte in Reaktion auf die jüngsten Versuche, Vers und Prosa einander anzunähern, eine drucktechnische Darbietungsweise, die in der Lage war, derartige Experimente künftig zu unterbinden. Die Leistung der von ihm propagierten wunsichtbaren Mittelachse« liegt demnach primär darin, die Grenze zwischen den konkurrierenden Ausdrucksmodi auf typographischem Wege wirkungsvoll zu reetablieren. ${ }^{41}$ Das schließt - obwohl Holz' Erfindung eine Reaktion auf die Einführung dieses Gattungsmusters in Deutschland darstellt - die Existenz von Prosagedichten natürlich keineswegs aus, verweist die neue Form aber klar in den Bereich der (unkünstlerischen) Prosa. „Revolution der Lyrik» darf sich dieser Kunstgriff deshalb nennen, weil dadurch die Lyrik als Vertextungsweise wieder trennscharf von ihrem prosaischen Widerpart geschieden war. ${ }^{42}$ Zugespitzt ausgedrückt: Wären alle Kollegen dem Vorschlag des Berliner Autors gefolgt, wären Mischformen wie die Spielarten der spoetischen Prosa fortan unmöglich und textuelle Subversionsexperimente wie das Prosagedicht zumindest aus dem Bereich der Lyrik verbannt gewesen. Erst auf Grund dieser einschneidenden Konsequenzen wird verständlich, wieso Holz sich für den grundlegenden "formalen Erneuerer der modernen deutschen Poesie* ${ }^{43}$

sönlichen Umgang beider hervorgegangen, wohnte doch Emst in den Jahren 1895 bis 1897 im Haus des jung verheirateten Holz und nahm in dessen Haushalt sogar regelmäßig die Mahlzeiten ein; vgl. Paul Ernst: Jünglingsjahre. München: Georg Müller 1931, S. 313. Im Sommer 1897 entbrannte zwischen beiden ein Streit um die Erstpublikation der optisch neuartig gegliederten Texte (vgl. W X, S. 401-444). Wie einem Schreiben Dehmels an Bierbaum vom 13. Juli 1893 zu entnehmen ist, machte Holz ersteren schon vor der Erstveröffentlichung mit Schlafs Frübling bekannt. Am 12. Juli habe Dehmel dann den Text im Beisein von Holz vorgelesen; vgl. Richard Dehmel: Brief an Bierbaum. In: Modemer Musen-Almanach auf das Jahr 1894, S. 271.

41 Der Autor mußte dazu, wie der Verzicht auf die konventionelle Großschreibung des Zeilenanfangs zeigt, nicht einmal Anleihen bei der herkömmlichen Verslyrik machen. Schon Servaes weist deshalb mit Recht die Ansicht »thörichter Recensenten«, wonach im Phantasus "Formlosigkeit herrscht", zurück und hebt den wdurchgebildeten Formeninstinct" (MuD-J, S. 36 und 39) Holz' hervor.

43 Amo Holz an Karl Hans Strobl, 25.6.1900; Amo Holz: Briefe, S. 127. In der Befriten deutschen Wortkunst (1918) heißt es dann nicht weniger unbescheiden: Die "[...] deutsche Wortkunst [...] wurde durch mein Ringen, dessen grundlegende Dokumente, praktisch und theoretisch, schwarz auf weiß vorliegen, im entscheidenden Durchbruch [...] befreit und wird nun [...] formal, und damit natürlich nicht bloß formal, die Weltliteratur endlich befrucheen, nachdem sie bisher, formal, und damit natürlich nicht blo $\beta$ formal, nur immer von ihr befruchtet wurdel« (W X, S. 638) Gegen Holz' Selbsteinschätzung spricht auch nicht, daß im Phantasus später malle vorher so sehr verworfenen traditionellen Formelemente, Metrum, Reim usw. als sogenannte sekundäre Stilmittek wieder neu aufgenommen« werden; Karl Geisendörfer: Die Entwicklung eines lyrischen Weltbildes im Pbantasus von Amo Holz, S. 246. Denn letztlich ist eben die Refunktionalisierung der »Bindemittel der traditionellen 
halten konnte. Was auf den ersten Blick wie ein einzigartiger textueller Entgrenzungsversuch erscheint, ${ }^{44}$ erweist sich bei eingehender Analyse mithin als im Kern konservative, nämlich auf die Stabilisierung des bisherigen Literatursystems ausgerichtete Maßnahme. ${ }^{45}$

Damit war eine Ausdrucksform von potentiell unendlicher Variabilität gefunden, ${ }^{46}$ die es möglich machte, jede Verszeile absolut individuell und einzigartig zu gestalten, ohne auf eine klare Abgrenzung von der Prosa verzichten zu müssen. Das literarische Kunstwerk erhält so eine sfließender Struktur, die auch durch den Seitenwechsel nicht wirklich unterbrochen wird, weil sie sich ja auf den vorhergenden und folgenden Blättern fortsetzt. Ziel dieser optischen Aufhebung des Textrahmens ist letztlich aber nicht Gattungsentgrenzung, ${ }^{47}$ sondern Entbindung der "Wortkunst« auf der Basis eines gesicherten Poetizitätsstatus. Versdichtung wird so zu einem

Lyriku nach 1916 nur Ausdruck auktorialer Souveränität, da der Einsatz der einst überwundenen Ausdrucksmittel ja im Rahmen seines Mittelachsensystems erfolgt; ebd., S. 247.

So meint etwa Geisendörfer: „Der Phantasus als Ganzes kann [...] als ein nicht näher eingrenzbarer Versuch gedeutet werden, alle verfügbaren sprachlichen Ausdrucksmöglichkeiten, vom kunstvollsten Vers bis zur einfachsten Prosa, zu einer einzigen Aussageform zusammenzupressen. « Ebd., S. 248.

Dies entgeht Frels' sehr vordergründig auf gesellschaftliche Ideologiekritik ausgerichteter Darstellung; vgl. Onno Frels: Zum Verhälmis von Wirklichkeit und künstlerischer Form bei Amo Holz. In: Naturalismus/Ästhetizismus. Hrsg. von Christa Bürger, Peter Bürger und Jochen Schulte-Sasse. Frankfurt a.M.: Suhrkamp 1979 (= edition suhrkamp 992; Hefte für kritische Literaturwissenschaft 1), S. 103-138. Dabei hat Holz selbst in seiner Nexen Wortkunst beklagt, "daß man bis jetzt aufu vielen "Gebietenı der Kunst "zu radikal mit den betreffenden iTraditionen gebrochen habe, was zu "Chaosk und "Zuständen rein negativer Natur" gefüht habe. "Neue Integrationsstufen, die Naturwissenschaft lehrt und beweist das, bilden und bauen sich aber nicht dadurch, $\mathrm{da} \beta$ sie die ihnen voraufgegangenen zertrümmem und auflösen, sondem vielmehr einzig dadurch, daß sie diese alle in sich zu höberen Einheiten begreifen!" Seine rhythmusbasierte Mittelachsenlyrik versteht er denn auch anders als die Zeitgenossen - ausdrücklich als »Positivum ( $(W X, S .721$ ), das er den verderblichen literarischen Formauflösungstendenzen seiner Zeit entgegengesetzt.

Wie Holz in seiner Schrift Die newe Wortkunst schreibt, ging es ihm ausdrücklich darum, munregelmäßig abgeteilte Zeilen« (W X, S. 623) zu erzeugen. Die angestrebte Vermeidung von Wiederholungsstrukturen auf der optischen Ebene bewirkt jenen Zwang zur Variation, der für den Autor dann Motor der literarischen Produktion wird.

47 Immerhin entgrenzt Holz den Begriff des Lyrischen, indem er - durchaus zeittypisch seinen Geltungsbereich über die Grenzen der Literatur hinaus ausdehnt. Seiner Ansicht nach kann man jedenfalls "Lyriker sein zum Beispiel auch als Maler und Musiker» (W X, S. 605). Auch überträgt der Autor ohne zu zögem Begriffe aus der Dramatik auf die Lyrik; so bemerkt er über die einzelnen Abschnitte seines lyrisches Hauptwerks: "Das [...] Anfangsstück, trotz seiner scheinbaren, nur rein äußerlichen Dreiteilung, ist ein 'Einakter, das große resümierende Schlußstück ein 'Dreiakter, das im Phantasus figurierende zweite Stück [...] gibt sich [...] als Siebenakter und so weiter, und so weiter!« (W X, S. 663f.) Vgl. dazu abermals Helmut Koopmann: Entgrenzung. Zu einem literarischen Phänomen um 1900. 
Analogon von Natur: ${ }^{48}$ Genau wie das naturhafte Sein sich durch alle Bereiche des Ontischen hindurch erstreckt, so läuft auch das nicht mehr von ästhetischen Konventionen eingeengte Textgebilde scheinbar ins Unendliche weiter. ${ }^{49}$

Gleichwohl ist Holz' Mittelachsengliederung ein typographisches Darstellungsverfahren, das nur in einer hochentwickelten Schriftkultur - einem "papierenen Zeitalter" (W X, S. 676) - denkbar ist. Borchmeyer konstatiert denn auch mit einem gewissen Recht, daß damit wein neues - visuelles Ordnungsprinzip der Lyrik eingeführt " ${ }^{50}$ worden sei. Der Autor formt also die Gattungszugehörigkeit eines Textes $z u$ einem optischen Superzeichen. Seine Gedichte leben geradezu von ihrer typographischen Gestalt; ${ }^{51}$ sie rücken das Element der Lautlichkeit, dem wegen der Bedeutung des $>$ Rhythmischen durchaus eine zentrale Bedeutung zukommt, zwar nicht in den Hintergrund, ordnen es aber dem Druckbild nach. ${ }^{52}$ Holz generiert auf diese Weise ein Modell, das Lyrik nicht auf eine obsolet gewordene Performanzebene zurückführt, sondern sie als graphische Struktur ernstnimmt; ${ }^{53}$ ja in gewisser Weise substituiert er Phonetik durch Graphemik, läßt aus Wortmusik bildliche Wortornamentik werden. ${ }^{54}$

48 Dies verbindet Holz' Phantasus-Ästhetik dann doch mit Schlafs Theorie vom mnatürlichen Rhythmus« (vgl. Kapitel III/4b).

Das Kunstwerk wird letztlich biomorph verstanden. So zeigt Holz sich davon überzeugt, es werde ihm mit dem Phantasus gelingen, waus tausend Einzelorganismen nach und nach einen riesigen Gesamtorganismus zu bilden, der lebendig aus ein und der selben Wurzel wächst« (W X, S. 652).

50 Dieter Borchmeyer: Der Naturalismus und seine Ausläufer, S. 206. Visuell war freilich - genau besehen - schon das alte, doch hat dies vor Holz kaum jemand zum Gegenstand der Reflexion gemacht.

Und so wurde der Autor, dem es doch in erster Linie auf die rinnere Form ankam (vgl. Kapitel III/7), in den Augen der Zeitgenossen zumeist als "Künstler der Außenform» (W) $\mathrm{X}, \mathrm{S} .507$ ) wahrgenommen.

52 Im Hinblick auf den Schlüsselbegriff von Holz' Poetik kann man in diesem Zusammenhang auch von einer literarischen Artikulation auf zwei Ebenen sprechen: wa rhythm that was perceptible to both ear and eye«; Rob Bums: The Quest for Modemity: The Place of Amo Holz in Modern German Literature, S. 162. Der Autor selbst versuchte den optischen Charakter seiner Texte freilich ein Stück weit zu relativieren und pries sein Verfahren metaphorisch vollmundig als "typographische Musik«, die das "Obrbild eines Gedichtes» (W) $X, S .659$ ) erzeuge.

53

Er hat damit durchaus teil am Typus arabesker Kommunikation, wie er im europäischen Ästhetizismus prägend geworden ist; vgl. Annette Simonis: Literarischer Ästhetizismus. Theorie der arabesken und hermetischen Kommunikation der Modeme.

54 Wenn Holz im Gegensatz dazu die Ansicht vertrit, daß die Texte, mum ihre volle Wirkung zu üben, den lebendigen Vortrag verlangten« (W X, S. 503), dann dienen solche Aussagen in erster Linie dazu, seine Innovation an die klassischer Lyriktheorie mit ihrem Primat der Mündlichkeit anzukoppeln. Emrich macht sich deshalb unreflektiert zum Apologeten des Autors, wenn er fordert: "Der Phantasus darf nicht gelesen, er muß sorgfältig nach den Angaben des Dichters gesprochen werden. Dann erst erschließt sich seine hohe dichterische 
So phantastisch die Pbantasus-Dichtung durch ihre die Raum/Zeit-Koordinaten sprengenden und die Subjekteinheit eines kohärenten lyrischen Ich auflösenden Tendenzen im einzelnen auch wirken mag, eine wirklich autonome Sprachkunst, die sich bereitwillig dem sfreien` Spiel der Signifikanten überläßt, ${ }^{55}$ entsteht nicht. Das verhindert die ontologische Fundierung von Holz' Ästhetik. Wie bedenkenlos der Berliner Autor Kunst und Natur kurzschloß, zeigt der Umstand, daß er - die briefliche Anregung von Robert Reß, einem Bewunderer seiner Texte, ${ }^{56}$ aufgreifend schließlich sogar eine uprinzipielle [...] Strukturanalogie [...] zwischen den drei Grundformen von Materie und den [...] drei Grundformen von Wortkunst« (W X, S. 729) postulierte:

Die amorphe Materie, gleichgültig ob gasförmig, flüssig oder fest, Zustände, die tatsächlich gar nicht scharf voneinander abgegrenzt werden können, ist in der Anordnung ihrer Atome resp. Moleküle, sowie in deren Bewegung eine im Prinzipr völlig regellose oder chaotische; weshalb sie denn auch keine individuell gesetzmäßig bestimmte Gestalt oder Begrenzung zu zeigen vermag. Sie ist somit unter allen drei genannten Gesichtspunkten die Unform der Materie. Desgleichen ist aber auch die Prosa, unter dem hier einzig in Betracht kom-

Kunst.« Wilhelm Emrich: Amo Holz und die modeme Kunst, S. 162. Dem steht freilich eine Erfahrung wie die Rilkes entgegen, der über dieses Werk bemerkt: »Hört man die Verse lesen, so [...] vernimmt [...] [man] eine bunte, teilweise unklare Prosa, in welcher dann und wann eine Allitteration [sic] oder eine onomatopoetische Verbindung auffallt, oder durch Wiederholungen eine Störung bewirkt wird. (R-SW V, S. 383) Nur das Sprechen des Textes beim Lesen, nicht aber das bloße Hören, entbirgt also dessen optische und akustische Dimension gleichermaßen.

55 Nach Brandstetter minstalliert Holz die Syntax selbst, mittels eines ihr verpflichteten Druckbilds als Gestaltungsprinzips; Alois Brandstetter: Gestalt und Leistung der Zeile im Phantasus von Amo Holz. Ein Beitrag zur Ästhetik der Syntax. In: Wirkendes Wort 16 (1966), S. 18. Es geht freilich zu weit, von der »Autonomie syntagmatischer Organisation« zu sprechen; ebd. Eher ließe sich sager, daß Holz linguistische Bauprinzipien der deutschen Syntax und Grammatik optisch sinnfallig hervorhebt, wodurch der Eindruck erweckt wird, diese Ordnung sei nicht nur logisch, sondem auch zwangsläufig. Schultz hat an Textbeispielen gezeigt, wie der Autor aus Sätzen mit gleichförmigem Betonungsmuster »durch die Gliederung des Wortmaterials in verschieden lange, durch kurze Pausen begrenzte Abschnitte einen äußerst spannungsreichen Rhythmus, der keinen permanenten Wechsel von Hebung und Senkung kenntu, erzeugt; Hartwig Schultz: Vom Rhythmus der modemen Lyrik. Parallele Versstrukturen bei Holz, George, Rilke, Brecht und den Expressionisten. München: Hanser 1970 (= Literatur als Kunst), S. 101. Letztlich gilt deshalb: Die "Autonomie des Rhythmus gibt Holz zugunsten seiner Semantisierung preis«; Dieter Borchmeyer. Der Naturalismus und seine Ausläufer, S. 206.

Überhaupt gab es eine Reihe von Autoren, die Holz' Mittelachsengliederung übernahmen oder imitierten; neben Paul Emst und den Autozen des Holz-Kreises Rolf Wolfgang Martens, Reinhard Piper, Robert Reß sowie Georg Stolzenberg waren dies etwa Paul Victor und Emil Alfred Hermann. Selbst Richard Dehmel hat, "vermutlich von seinem damaligen Freunde Holz inspiriert", Versgedichte in Weib und Welt (1896) wie auch einige Texte von Zuei Menschen (1898) achsenzentriert drucken lassen; Gerhard Schulz: Amo Holz. Dilemma eines bürgerlichen Dichterlebens, S. 76. 
menden Gesichtspunkte, nämlich dem wortkünstlerischen, eine innerlich wie äußerlich völlig ungebundene, also die «Unformx der Wortkunst. (W X, S. 723)

Diesem ästhetisch minderwertigen, ungeordneten Zustand stellt Holz dann die herkömmliche, "streng gleichförmige "Versmetrik entgegen, die der »kristallinen Form der Materie« (W X, S. 724) entspreche. Weit überboten werde deren starre Ordnung aber noch von der rhythmischen Phantasus-Lyrik, die in ihrer Individualität und "permanenten Abdifferenzierung [...] sowohl der Struktur wie der Funktion« das Äquivalent zur "lebendig organisierten Materie» (W X, S. 725) darstelle. Indem die Gesetze der materiellen Welt auch als in der Ästhetik gültig gedacht werden, ergeben sich für die Dichtkunst fixe Organisationszustände bzw. Existenztypen, die ein für allemal vorgegeben und dem historischen Wandel entzogen sind. Und dadurch, daß die einzelnen "Strukturformen" im evolutionsbiologischen Sinn als zeitlich evolvierend gedacht werden ${ }^{58}$ stellt sich auch eine festgelegte Rangfolge der einzelnen "Entwicklungsetappen" (W X, S. 730) her: An unterster Stelle steht dabei die Prosa (»Unform(s), über ihr rangiert die prosodisch geregelte Verslyrik (")symmetrische [d.h. äußere] Form«), und die Spitze dieser Differenzierungsskala nimmt die von Holz eingeführte "Phantasushythmik» (W X, S. 722) (masymmetrische [d.h. innere] Form«) ein.

Entgegen der Ansicht vieler Zeitgenossen, die der Meinung waren, Holz hätte die Lyrik 'prosaisiert" phantastische sinnliche Prosau (R-SW V, S. 383), und Heinrich Hart bezeichnet den Phantasus als unglückliche "Versprosa-Erfindung" (W X, S. 555), die letztich nur "mühsam verkleidete Prosa" (W X, S. 556f.) biete -, wird Literatur bei ihm wieder auf $\gg$ Dichtung eingegrenzt ${ }^{60}$ und damit gewissermaßen ein anfänglicher Zustand vor der funktionalen Ausdifferenzierung der Formen restituiert. ${ }^{61}$ Der Autor wirkt so

57 Abgesehen davon, daß ein solches Analogiedenken nichts anderes ist als haltlose Spekulation, unterminiert Holz die Ernsthaftigkeit seiner Argumentation schon allein durch eine völlige Unkenntris physikalischer Sachverhalte. Auch um 1900 war längst bekannt, daß der physikalische Gestaltcharakter eines Stoffes entscheidend von seinem Aggregatszustand abhängt, es eine mamorphe Materie» an sich mithin nicht gibt.

"This idea of development, that is to say, the process of change but within a framework of continuity, was crucial to Holz, so much so, in fact, that he altered the title of his script of 1899 from Revolution der Lyriks to 'Evolution der Lyriks in order to emphasize that he was concerned not so much to make a radical break with tradition as to develop usefully what was rooted in it.، Rob Burns: The Quest for Modernity: The Place of Amo Holz in Modern German Literature, S. 146. In seiner Nexen Worteunst weist Holz denn auch den Einwand, sein mneuer [...], notwendiger Rhythmus [...] wäre nur Prosax, weshalb er die Texte mauch als solche schriben, beziehungsweise drucken lassen (W X, S. 658) solle, mit Entschiedenheit ab.

60 Burdorf bemerkt in diesem Zusammenhang zu Recht: „Holz betreibt also die Einebnung der Gattungsgrenzen zugunsten einer umfassenden Lyrisierung aller Dichtung im Zeichen der sneuen Formu; Dieter Burdorf: Poetik der Form. Eine Begriffs- und Problemgeschichte, S. 374, Anm. 28.

61 Dies verbindet ihn abermals mit Schlaf, der freilich annimmt, die Prosa (bzw. ein Konglo- 
nicht nur der zunehmenden Aufweichung des Gattungssystems machtvoll entgegen, er lenkt auch die 'Bedrohung der Lyrik durch Genres wie das Prosagedicht geschickt ab. Holz kann mithin als Protagonist jener Fraktion von Schriftstellern gelten, die das Terrain der Versdichtung dauerhaft gegen textuelle Subversionsexperimente zu sichern suchen.

An Holz' Beispiel zeigt sich im übrigen noch einmal sehr deutlich, wie unterschiedlich die Rahmenbedingungen für die Etablierung und Ausbreitung des Prosagedichts in den einzelnen europäischen Nationalliteraturen faktisch waren. Anders als in Frankreich, wo das poème en prose seine Blüte im dezidiert lyrikfreundlichen Kontext des Ästhetizismus und des l'art pour l'art erlebte, fand die Erstrezeption des Genres hierzulande im Zeichen der - zumindest von ihrem Selbstverständnis her - am Ausdrucksmodus der Prosa orientierten naturalistischen Bewegung statt. Dieser Ausgangslage ist es denn auch zuzuschreiben, daß die Form, kaum daß sie in Deutschland einigermaßen etabliert war, umgehend unter Legitimationsdruck geriet. Der Grund dafür liegt in der Differenz der ästhetischen Entwicklung. Der Umstand, daß der deutsche Naturalismus nur vergleichsweise kurze Zeit seine Stellung als literarisches Leitparadigma aufrechterhalten konnte und bereits nach wenigen Jahren von gegennaturalistischen Strömungen abgelöst wurde - ein Trend, dem im übrigen selbst seine Verfechter keinen nennenswerten Widerstand entgegensetzten -, ließ die von ihm bevorzugten (oder auch nur mit ihm assoziierten) Ausdrucksformen und Gattungsmuster rasch überholt, ja sogar schädlich wirken. Während in Frankreich die normsprengende Funktion des Prosagedichts vor dem Hintergrund der hartnäckigen Persistenz eines klassizistisch geprägten Systems künstlerischer Formen fast durchweg positiv aufgenommen wurde, verlief in Deutschland die Wahrnehmung der vom Naturalismus propagierten Außerkraftsetzung poetischer Konventionen überaus ambivalent. Das damit einhergehende Aufbrechen des bisherigen Kanons literarischer Ausdrucksformen erschien nicht automatisch als Befreiung, sondern zugleich auch als latente Bedrohung der nach wie vor auratisierten Verslyrik.

Der Abbau von Vertextungsnormen hatte zwar zu einer Entgrenzung des Formenkanons geführt, doch war es nicht gelungen, die ästhetische Wertskala literarischer Gattungen im gleichen Zug nachhaltig zu verändern. Auch wenn die Naturalisten die Prosa vollmundig zum einzig modernen Gestaltungsregister erklärt hatten, hatte sich von den Vertretern dieser Bewegung kaum jemand dazu bereitgefunden, sie wirklich ernsthaft zu privilegieren. Der Grund für diese inkonsequente Haltung liegt auf der Hand: Der im Vergleich mit den konkurrierenden Gattungen deutlich größere ökonomische Erfolg der Erzählprosa (das Versepos spielt hier eine Sonderrolle $^{62}$ ), der gleichermaßen aus ihrer leichteren Rezipierbarkeit und ihrer vielseitigen

merat von Prosarede, Gestik und Tanz) sei der ursprüngliche Ausdrucksmodus gewesen; vgl. Kapitel III/4b.

62

Vgl. Das deutsche Versepos. Hrsg. von Walter Johannes Schröder. Darmstadt: Wissen- 
Einsetzbarkeit resultierte, hatte in den Köpfen der Leser und Autoren nachhaltig ein Wahrnehmungsstereotyp verankert, wonach alle narrativen Genres als überwiegend kommerzielle, auf Verkäuflichkeit ausgerichtete und daher notgedrungen massenkompatible, d.h. auf die Rezeptionserwartungen des Publikums zugeschnittene Textformen erschienen. ${ }^{63}$ Dieser generelle Verdacht kompromittierte alle Prosaformen nachhaltig, und mit ihnen die prosanahen lyrischen Gattungen gleich mit.

Liliencrons Texte beispielsweise riefen nach dem überwältigend positiven Echo, das sie bei den Naturalisten gefunden hatten, mit der Zeit mehr und mehr kritische Stimmen hervor, die an ihnen gestalterische Nachlässigkeiten bemängelten. In Hans Paulis Aufsatz Detlev von Liliencron wird dem Autor gar Anbiederung an journalistische Verwertungspraktiken vorgeworfen:

Im Liliencron [...] ist eine Seite, die sich immer mehr feuilletonisiert hat. Seine Liebe zu der Form der Ottave rime, die die buntesten Eskapaden erlaubt, wurde immer grösser, und dieselbe Veranlagung liess ihn sich in freien Rhythmen tummeln. [...] Aber Liberté oblige [...]. Bei Liliencron sind die freien Verse nun nicht grade tollgewordene Prosa, aber doch Prosa, die nur Poesie von Druckers Gnaden ist. [...]

Was ich meine ist dieses: In Liliencrons Produktion ist eine, in den Adjutantenritten fast gar nicht zu spürende, später mehr hervortretende Hinneigung zum poetischen Feuilleton, beziehungsweise zu improvisatorischer Behandlung. [...] Ohne gerade salopp zu werden, liess er sich gehen. ${ }^{64}$

Rudolf Borchardt dann wandte sich schlechterdings gegen alle Arten von Mischgattungen und meinte in der Modeme nichts anderes als zur Formlosigkeit ${ }^{65}$ tendierende "Anarchie ${ }^{66}$ zu entdecken:

schaftliche Buchgesellschaft 1969 (= Wege der Forschung 109) und Nicole Ahlers: Das deutsche Versepos zwischen 1848 und 1914. Frankfurt a.M./Berlin/Bern/New York/Paris/Wien: Lang 1998 (= Hamburger Beiträge zur Germaniscik 26).

So erklärte der Amo-Holz-Epigone Paul Emst in seinem Aufsatz Ethische Kultur (1899) kategorisch: "Der Roman wird stets Halbkunst bleiben, weil ihm der Zwang zur Form fehlt." (MuD-J, S. 427) Und auch für die Folgegeneration ist der Roman noch fraglos die "für Deutschland von altersher [...] am wenigsten ergiebige und unselbständigste Kunstformu; Emst Stadler: Dichtungen, Schriften, Briefe. Kritische Ausgabe. Hrsg. von Klaus Hurlebusch und Kaul Ludwig Schneider. München: C.H. Beck 1983, S. 325.

Neue deutsche Rundschau 7 (1896), S. 788.

65 Bedeutsam ist in diesem Zusammenhang, daß mit Nietzsche der einflußreichste ideologische Stichwortgeber der Zeit in Menschliches, Allzumenschliches (1878) den "Naturalismus" in der Ästhetik als Abkehr von der Form gedeutet hat; vgl. Friedrich Niezsche: Sämtliche Werke, Bd. 2, S. 181. Er trug so entscheidend dazu bei, daß die Rede von der Formlosigkeit des Naturalismus - und folglich auch der Prosa - um 1900 endemisch wurde.

66 Rudolf Borchardt: Rede über Hofmannsthal [1902/07]. In: Gotthart Wunberg/Stephan Dietrich (Hrsg): Die literarische Modeme. Dokumente zum Selbstverständnis der Literatur um die Jahrhundertwende. 2, verbesserte und kommentierte Auflage. Freiburg i.Br.: Rombach 1998 (= Rombach Wissenschaften - Reihe Litterae 60), S. 231. 
Sie haßt jene Notwendigkeit, die Form, jede Strenge, die Gattung, jede Reinheit, den Typus. Sie schafft verwischte Form und nennt sie Nuance, zerrissene Form und nennt sie Skizzen, Gedichte in Prosa, Freie Rhythmen, - Zwittergattungen, und nennt sie DDokumente, ,Impressionen, , Phantasien, ,Stimmungen, , Tragikomödie ${ }^{67}$

Derartige Akte poetologischer Abgrenzung delegitimierten die Spielarten ungebundener Rede weitgehend, so daß die Prosa im Laufe der neunziger Jahre ihren bis dahin weitverbreiteten Nimbus als flexibles und modernes، Gestaltungsmittel einbüßte und zunehmend als gleichermaßen unökonomischer wie ästhetisch ineffizienter literarischer Ausdrucksmodus wahrgenommen wurde. Im Gegenzug erfuhr die formbewußte, auf sprachliche Exklusivität ausgerichtete Verslyrik eine deutliche Aufwertung; die vermeintliche Marktferne und der ihr gern unterstellte, latent solipsistische Charakter verliehen ihr überraschend eine neue, glanzvoll wirkende Aura. ${ }^{68}$

Es kam deshalb bereits vor der Jahrhundertwende zu einer massiven Revitalisierung der Versdichtung. Schon 1890 hatte Julius Hart, einer der ehemaligen Wortführer der naturalistischen Bewegung, die Überzeugung geäußert, die Literatur befinde sich in einer Entwicklung, die wvon der Prosa zum Verse wieder emporführen wird (MuD-N, S. 139). Er appellierte deshalb an seine Kollegen, die bisherige »Feindschaft gegen den Vers» (MuD-N, S. 133) zu beenden, und forderte eine baldige Rückkehr zur Verslyrik:

Die höchste Macht des Empfindens und der Phantasiekraft bedarf der Aussprache durch den Vers. [...] Wir können bei dieser Frage mit vollem Recht auf das Gesetz vom kleinsten Kraftmaß zurückgreifen. Durch viel geringere Mittel erreicht der Vers doch besser und unmittelbarer das Ziel der Gefühls- und Phantasieerregung, als es die dichterische Prosa vermag. [...] Der Vers wirkt aber deshalb so unmittelbar, weil Inhalt des Wortes, Klang und Rhythmus sich völlig durchdrungen haben, weil alle drei Faktoren zu gleicher Zeit auf unsere Phantasie und unser Gefühl zustoßen, während die Prosa vorwiegend nur durch ihr Inhaltliches anregt. (MuD-N, S. 138)

Und selbst ein Autor wie Wilhelm Bölsche sprach sich nun nachdrücklich dafür aus, die Literatur wieder als Gegenpart zur Alltagsrede zu verstehen, welche nur »nacktes Kommunikationsmittel ${ }^{69}$ sei, und scheute nicht einmal davor zurück, offen für eine versifizierte Bühnensprache zu plädieren. Die Entscheidung, die der moderne Künstler zu treffen habe, stelle sich folgendermaßen dar: Entweder

[...] Wiederholung unserer konventionellen Verkehrssprache auch auf der Bühne, dieser Verkehrssprache, die mit jedem Tage mehr abgeschliffen wird und aus reinen Nützlich-

67 Ebd., S. 232

68 Žmegač bemerkt zu Recht, daß die »Opposition ,Vers - Prosar [...] um 1900 zunehmend mit den Konnotationen rästhetisch verfeinert, autonom, idealistisch, versus mimetisch, stoffgebunden, materialistisch« verknüpft werde; Viktor Żmegač: Kunst und Ideologie in der Gattungspoetik der Jahrhundertwende [1980], S. 90.

69 Wilheim Bölsche: Eine Lanze für den Vers im Drama. In: Die Gesellschaft 17 (1901), Bd. 2, S. 42 . 
keitsgründen jedes Sonnenstäubchen Schönheit ausmerzt, - oder [...] fort und fort bewährte Pflege jener anderen Wurzel der Sprachentwicklung überhaupt, die von der Ästhetik, von der Schönheit, von der Harmonie kommt. ${ }^{70}$

Der Ansicht Harts und Bölsches schlossen sich - mit individuellen Modifikationen die meisten Kollegen an. Und so konnte ein Autor wie Carl Busse, der noch im selben Jahr mit Prosagedichten hervortrat, ${ }^{71} 1896$ eine neue, nämlich die »dritte Phase» der Moderne ausrufen, die er durch ndas Streben nach einer künstlerischen Form ${ }^{72}$ gekennzeichnet sah.

Tatsächlich lassen sich "formrestaurative Tendenzen ${ }^{73}$ um 1900 in allen Sektoren des Gattungssystems erkennen. Die zeitgenössische Narrativik etwa ist gekennzeichnet durch das »Bestreben, der als Formzerfall gedeuteten naturalistischen und impressionistischen Prosa mit der Erneuerung fester, geschlossener Erzählungstypen zu begegnen «, was u.a. zur "Wiederbelebung der Novelle ${ }^{74}$ klassisch strenger Machart führt. Ähnlich begegnen auch in der Lyrik mannigfaltige Versuche, herkömmliche formale Gestaltungsmittel zu restituieren: „Das Bedürfnis nach regelmäBiger Vers- und Strophenanordnung, einem alternierenden Rhythmus und traditionellen Reimschemata verdrängte die ursprünglichen Stilmittel der neunziger Jahre des vergangenen $[=19$.$] Jahrhunderts. \kappa^{75}$ Dennoch kann von einer $»$ Ablösung der freien Dichtungsformen ${ }^{76}$ keine Rede sein. Obwohl es zu dieser Zeit tatsächlich einen zumindest partiellen »Rückbezug auf konventionelle» Darstellungsmuster gibt, bedeutet das noch nicht, daß damit eine neue Phase der "Formstrenge ${ }^{7}$ angebrochen ist. Vielmehr kommt es zu einer veränderten Einschätzung dessen, was als zzeitgemäßes Poetizitätssignal zu gelten habe. Dabei erleben zwar einige traditionelle verstechnische Verfahrensweisen eine Neubelebung, zugleich bleiben aber auch zahlreiche Lizenzen der lyrischen Gestaltung, die sich seit dem Naturalismus durchsetzen konnten, exhalten, ja bestimmte Standards des ästhetischen Normbruchs (gemessen an der Zeit vor 1880) erweisen sich geradezu als unabdingbarer Ausweis der eigenen ıModernität. Offenbar wird in den neunziger Jahren des 19. Jahrhunderts das Verhältnis von 'Tradition und Innovation im Bereich der Lyrik neu austariert. Einer der tragenden Impulse besteht dabei unzweifelhaft darin, der von der

70 Ebd., S. 44.

71 Vgl. hierzu Kapitel III/8.

72 Karl [sic] Busse: Die modeme Bewegung in der deutschen Literatur, S. 858.

73 Viktor Žmegač: Kunst und Ideologie in der Gattungspoetik der Jahrhundertwende [1980], S. 90.

74 Ebd.

75 Elisabeth Veit: Fiktion und Realität in der Lyrik. Literarische Weltmodelle zwischen 1890 und 1918 in der Dichtung Max Dauthendeys, Richard Dehmels und Alfred Momberts, S. $139 f$. 
Moderne entbundenen gestalterischen Willkür Grenzen zu setzen und die Literatur zumindest teilweise wieder an bestehende Traditionen rückzubinden. Dies kann freilich nicht einfach dadurch geschen, daß feststehende Vertextungsregeln emeut für verbindlich erklärt werden. Die Suche, welche eine Vielzahl von Autoren um die Jahrhundertwende bewegt, richtet sich denn auch nicht mehr auf die räußere, sondern auf die sinnere Form der Texte. ${ }^{78}$ Damit wird die systemische Grenze zwischen >Poesier und Prosa nicht mehr länger auf die Dichotomie von sForme und IInhalt abgebildet, und dem Prosagedicht bleibt immerhin ein Gestaltungsreservat erhalten. Ästhetisch konkurrenzfähig ist es fortan allerdings nurmehr als überholten Traditionen nicht verhaftetes Modell freien spoetischen، Ausdrucks, nicht aber als Typus schmuckloser Prosarede. ${ }^{79}$ Und so sieht sich die Gattung in den kommenden zwanzig Jahren fast ganz auf den Bereich lyrisierender Prosa verwiesen.

Siehe hierzu Kapitel III/7.

79 Dem Druck zur Poetisienung literarischer Darstellungsformen sahen sich auch Prosagroßformen wie der Roman ausgesetzt. Der dänische Erzähler Jens Peter Jacobsen etwa wurde im deutschen Sprachraum vor allem deshalb begeistert rezipiert, weil er - wie sich ein Zeitgenosse ausdrückt - neinen neuen Lyrismus in die Prosa gebracht" (DJW II, S. 703) habe. 